\title{
Adapters in lymphocyte signaling
}

\author{
Albrecht Leo, ${ }^{1}$ Jürgen Wienands, ${ }^{2}$ Gottfried Baier, ${ }^{3}$ Vaclav Horejsi, ${ }^{4}$ and Burkhart Schraven ${ }^{5}$ \\ ${ }^{1}$ Institute for Immunology, Ruprecht-Karls-University Heidelberg, Heidelberg, Germany \\ ${ }^{2}$ Institute for Biochemistry, University of Bielefeld, Bielefeld, Germany \\ ${ }^{3}$ Institute for Medical Biology and Human Genetics, University of Innsbruck, Innsbruck, Austria \\ ${ }^{4}$ Institute of Molecular Genetics, Academy of Sciences of the Czech Republic, Prague, Czech Republic \\ ${ }^{5}$ Institute for Immunology, Otto-von-Guericke-University, Magdeburg, Germany \\ Address correspondence to: Burkhart Schraven, Institute for Immunology, Otto-von-Guericke-University Magdeburg, Leipziger Strasse 44, \\ D-39120 Magdeburg, Germany. Phone: 49-391-67-15800; Fax: 49-391-67-15852; E-mail: burkhart.schraven@medizin.uni-magdeburg.de.
}

J. Clin. Invest. 109:301-309 (2002). DOI:10.1172/JCI200214942.

In order to exert their effector functions, lymphocytes need to be activated. This process requires at least two stimuli, a primary stimulus, which is mediated via an immunoreceptor (e.g., the $\mathrm{T}$ cell receptor [TCR], the B cell receptor [BCR], or the Fc receptors [FcRs]), and a costimulatory signal (see Frauwirth and Thompson, this Perspective series, ref. 1 ), which is mediated via so-called accessory receptors (e.g., CD28 on T cells and CD19 on B cells). These two types of signals, as well many others that are generated by triggering of regulatory receptors expressed on the lymphocyte surface, are transmitted to the intracellular compartment, where they initiate cascades of biochemical events that finally produce a cellular response. How, then, are these externally applied signals integrated to yield an appropriate immune response?

Here we review the current knowledge about the membrane-adjacent signaling events that are generated immediately after triggering of immunoreceptors, focusing in particular on the functions of one group of signaling proteins, collectively termed "adapter" or "linker" proteins. In addition, we offer a (very subjective) discussion of key unanswered questions related to the connection of signal-transducing receptors with the cellular environment.

\section{TRAPs and CAPs}

By definition, adapter proteins lack both enzymatic and transcriptional activities but control lymphocyte activation by mediating constitutive or inducible proteinprotein or protein-lipid interactions via modular interaction domains. Such domains include, for example, Src homology 2 (SH2), Src homology 3 (SH3), phosphotyrosine-binding $(\mathrm{PTB})$, and pleckstrin-homology $(\mathrm{PH})$ domains, as well as tyrosine-based signaling motifs (TBSMs; for details regarding the binding specificities of these domains, see Figure 1). TBSMs comprise short peptide sequences containing a core tyrosine residue, which, upon phosphorylation, mediates a high-affinity interaction with SH2 or PTB domains. The specificity of this interaction is determined by the amino acids flanking the tyrosine residue.
Two groups of adapter proteins can be defined: adapter proteins that represent integral membrane proteins (transmembrane adapter proteins, or TRAPs) and adapter proteins that are preferentially located in the cytoplasm (cytosolic adapter proteins, or CAPs). Four TRAPs have been identified and cloned: LAT (linker for activation of T cells), TRIM (T cell receptor-interacting molecule), SIT (SHP-2-interacting TRAP), and PAG/Cbp (protein associated with glycosphingolipid-enriched microdomains/Csk-binding protein). The known TRAPs all have short extracellular domains, which (except perhaps for SIT) probably do not bind specific external ligands. In contrast to the CAPs, most of which possess both modular binding domains and TBSMs, the cytoplasmic domains of the TRAPs lack SH2 domains, SH3 domains, and other modular protein-protein interaction domains but contain multiple (up to ten) TBSMs. These sequences become phosphorylated by Src and/or Syk protein tyrosine kinases (PTKs) after triggering of antigen receptors (Figure 1).

The main function of TRAPs can be deduced based on these structural properties. After phosphorylation, TRAPs serve as anchors for the $\mathrm{SH} 2$ domains of intracellular signaling and effector molecules (e.g., phospholipases, lipid kinases, protein tyrosine kinases, protein tyrosine phosphatases, or CAPs such as Grb2, Gads, and SLP-76), thus targeting the latter to the plasma membrane. This allows the formation and nucleation of membrane-associated signaling scaffolds, which are required for the propagation of receptormediated signals to the intracellular environment (for examples, see Figures 2 and 3).

\section{Adapters in signaling through the TCR}

Among the best-characterized membrane-associated signaling scaffolds in $\mathrm{T}$ cells is the $\mathrm{Ca}^{2+}$-initiation complex, which comprises LAT (a TRAP), Gads and SLP-76 (both CAPs), the effector molecules PLC $\gamma 1$ and Itk (a PTK of the Tec family), and the Grb2/SOS complex, which activates $\mathrm{p} 21^{\text {ras }}(2)$. The current model for $\mathrm{Ca}^{2+}$ 


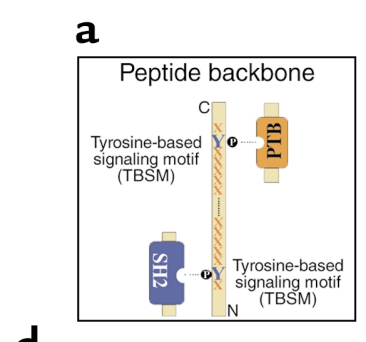

b

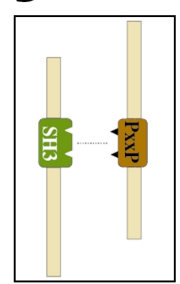

c

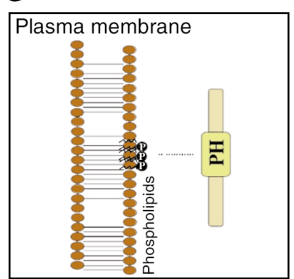

Name

Structure
$\mathrm{MW}(\mathrm{kDa})$ Expression

teins (TRAPs)

\section{Figure 1}

(a-c) Schematic illustration of the binding characteristics of the protein-protein or protein-lipid interaction modules expressed by adapter proteins. (a) In general, SH2 and PTB domains bind to phosphorylated TBSMs, whose binding specificities are determined by the amino acids surrounding the core tyrosine ( $\mathrm{C}$-terminal in case of PTB domains, $\mathrm{N}$-terminal in case of $\mathrm{SH} 2$ domains). (b) $\mathrm{SH} 3$ domains constitutively interact with prolinerich regions. (c) $\mathrm{PH}$ domains bind to phospholipids. (d) Schematic presentation of a selection of adapter proteins involved in proximal TCR signaling.

plasma membrane after phosphorylation (4). For example, the three C-terminal TBSMs of LAT ( $\mathrm{Y}^{171}, \mathrm{Y}^{191}$, and $\left.\mathrm{Y}^{226}\right)$ can mediate binding of Grb2 and its associated nucleotide exchange factor SOS (5). Which of these three tyrosines actually represents the major binding site for Grb2 in vivo is as yet unclear. Two of these TBSMs ( $\mathrm{Y}^{171}$ and $\left.\mathrm{Y}^{191}\right)$ can also facilitate binding of Gads (5), a CAP that associates constitutively with SLP-76. Binding of Gads to LAT therefore brings SLP-76 to the membrane $(6,7)$. Initially, it was thought that, by binding to LAT, SLP-76 might be brought into the vicinity of ZAP-70, thus allowing its phosphorylation (8). However, the most recent analysis of Gads-deficient mice suggests that phosphorylation of SLP-76 may be independent of binding to LAT (9). In any case, phosphorylated SLP-76 provides the binding site for the $\mathrm{SH} 2$

initiation complex formation and activation in $\mathrm{T}$ and B lymphocytes provides a good example of the mechanisms by which TRAPs operate in signal transduction (Figures 2 and 3 ).

Lymphocyte activation is initiated by triggering of immunoreceptors (TCR, BCR, FcRs) by their natural ligands (e.g., antigen/MHC in the case of $\mathrm{T}$ cells). Immediately thereafter, Src PTKs (Lck, Fyn, and Lyn) become activated by a mechanism that is still not completely understood, as discussed below, and in turn phosphorylate tandem tyrosine residues within specialized signaling motifs termed ITAMs (immunoreceptor tyrosine-based activation motifs; Billadeau and Leibson, this Perspective series, ref. 3). ITAMs are present in the cytoplasmic domains of the immunoreceptor-associated signal-transducing subunits such as $C D 3 \gamma,-\delta$, and $-\varepsilon$, the TCR $\zeta$-chains, and the BCR-associated signaling molecules $\operatorname{Ig} \alpha$ and $\operatorname{Ig} \beta$ (CD79a and CD79b). Dually phosphorylated ITAMs provide the docking sites for the tandem $\mathrm{SH} 2$ domains of the Syk family PTKs ZAP-70 (in T cells) and Syk (in B cells), which are thereby targeted to the cell membrane and subsequently become activated through phosphorylation by Src PTKs.

In T cells, one major substrate for ZAP-70 is LAT, which recruits several key signaling molecules to the domain of the tyrosine kinase Itk, to generate a membrane-associated complex consisting of LAT, Gads, SLP-76, and Itk (10).

Recruitment of Itk into this complex occurs in parallel with the binding of another key effector molecule, PLC $\gamma 1$, to one of the remaining TBSMs in LAT (5). Thus, PLC $\gamma 1$ is also integrated into the $\mathrm{Ca}^{2+}$-initiation complex, where it becomes activated by dual phosphorylation, mediated by ZAP-70 and Itk (Figure 3; see also ref. 11). Activated PLC $\gamma 1$ cleaves the membrane-associated phosphoinositide $\mathrm{PIP}_{2}$, thus generating the second messengers $\mathrm{IP}_{3}$ and DAG. $\mathrm{IP}_{3}$ causes mobilization of $\mathrm{Ca}^{2+}$ from intra- and extracellular stores, thus raising intracellular $\mathrm{Ca}^{2+}$ levels, whereas DAG represents a classical activator of conventional and novel protein kinase C (PKC) isotypes (Figure 2).

Among the PKC family members that are expressed in T cells, PKC $\theta$ stands out, since it seems to exert nonredundant functions that are essential for $\mathrm{T}$ cell activation. For example, PKC $\theta$ is required for activation of the transcription factors NF- $\mathrm{KB}$ and AP-1 $(12,13)$. Moreover, PKC $\theta$ appears to localize selectively into the center of the mature immunological synapse (IS; see Dustin, this Perspective series, ref. 14) during antigen stimulation (15). The elucidation of the molecular events underlying targeting of PKC $\theta$ to the IS is of par- 
ticular interest, especially because DAG is known to recruit all $\mathrm{PKC}$ isotypes to the plasma membrane.

Most recently it has been demonstrated that DAG also recruits the nucleotide exchange factor RasGRP (an activator of $\mathrm{p} 21^{\text {ras}}$ ) to the membrane (16). The identification of this novel pathway for $\mathrm{p} 21^{\text {ras }}$ activation may help to explain the observation that in Jurkat $\mathrm{T}$ cells lacking SLP-76, the association of LAT with the Grb2/SOS complex (the best-studied activator of p2 $1^{\text {ras }}$ is, by itself, not sufficient to activate p21 $1^{\text {ras }}$ (17). However, it is also not known whether binding of RasGRP to DAG alone is sufficient to activate $21^{\text {ras }}$, or whether DAG and the LAT/Grb2/SOS complex cooperate in its induction.

In $\mathrm{B}$ cells, essential components of the $\mathrm{Ca}^{2+}$-initiation complex include PLC $\gamma 2$, Btk (a Tec family PTK), and SLP-65, a CAP also known as BLNK. SLP-65 is clearly required for both the translocation of PLC $\gamma 2$ from the cytosol to the plasma membrane and its subsequent phosphorylation or activation. However, when expressed in SLP-76-deficient Jurkat T cells, SLP-65 cannot rescue TCR-mediated signaling. In marked contrast, expression of LAT, Gads, and SLP-76 rescues BCR signaling in an SLP-65-deficient B cell (18-20). The most plausible explanation for these surprising observations is that some still-unidentified TRAPs, perhaps related to LAT, act in B cells to bind SLP-65 and to nucleate the $\mathrm{Ca}^{2+}$-initiation complex, forming a structure similar to the LAT/Gads/SLP-76 complex in T cells. One experiment that could help to shed light on the function of SLP- 65 would be to test whether expression of a LAT-SLP- 65 chimera can rescue signaling in SLP-76-deficient Jurkat T cells.

Intriguingly, a recent study showed that the SH2 domain of SLP-65 can interact directly with a con- served TBSM in the cytoplasmic domain of Ig $\alpha$. Remarkably, this TBSM is located outside the ITAM (21). These data might indicate that BCR signaling subunits themselves can perform functions that, in T cells, are relegated to the $\zeta$-chain and LAT. If so, there may be no need for a LAT equivalent; Ig $\alpha$ itself could both bind Syk and mediate the formation of the $\mathrm{Ca}^{2+}$-initiation complex. However, very recent data from our laboratories (V. Horejsi et al., unpublished observations) indicate that a novel LAT-like protein does indeed play a role in BCR signaling.

\section{TRAPping Csk}

Given the continued uncertainties regarding the mechanism by which the TCR initially activates Src PTKs, recent hints that PAG plays a key role in this process are of great interest $(22,23)$. In contrast to previously described TRAPs, PAG is expressed as a constitutively tyrosine-phosphorylated protein in nonstimulated $\mathrm{T}$ cells, where it recruits the tyrosine kinase Csk, the major negative regulator of Src PTK, to the plasma membrane. The association between Csk and PAG is mediated through the $\mathrm{SH} 2$ domain of Csk and at least two TBSMs (B. Schraven, unpublished data) within the cytoplasmic domain of PAG. Thus, it seems as if one task of PAG may be to bring Csk to its substrates, the Src PTKs. The latter kinases are thereby inactivated through phosphorylation of their C-terminal negative regulatory tyrosine residues.

An attractive hypothesis emerging from this finding is that Src PTKs in resting T cells are subjected to tonic inhibition by Csk, which is trapped at the plasma membrane by constitutively tyrosine-phosphorylated PAG (24). Indeed, at least one of the two Csk-binding TBSMs of PAG becomes dephosphorylated after $\mathrm{T}$ cell activa-

\section{Figure 2}

Schematic illustration of the membrane-proximal signaling events in $B$ (left) and T cells (right). (î) Activation of raft-associated Src PTKs upon engagement of the immunoreceptor. (II) Recruitment of the BCR and TCR to the rafts. (III) Recruitment of Syk PTK to dually phosphorylated ITAMs. (d) Phosphorylation of members of the $\mathrm{Ca}^{+}$-initiation complex. (IV) Phosphorylationdependent assembly of the $\mathrm{Ca2}^{+}$-initiation complex. Note that it is not yet clear whether the $\mathrm{Ca}_{2}{ }^{+}$-initiation complex in B cells involves a LAT-like TRAP. (V) Generation of the second messengers $D A G$ and $I P_{3}$ via cleavage of $\mathrm{PIP}_{2}$ by activated PLC $\gamma$. (VI) Alteration of the actin cytoskeleton and regulation of adhesion and migration via SLP-76/SLP-65-associated adapter and effector molecules. (VII) Negative regulation of Src PTK activation via the TRAP PAG and the Src kinase inhibitor Csk.
BCR-mediated signaling

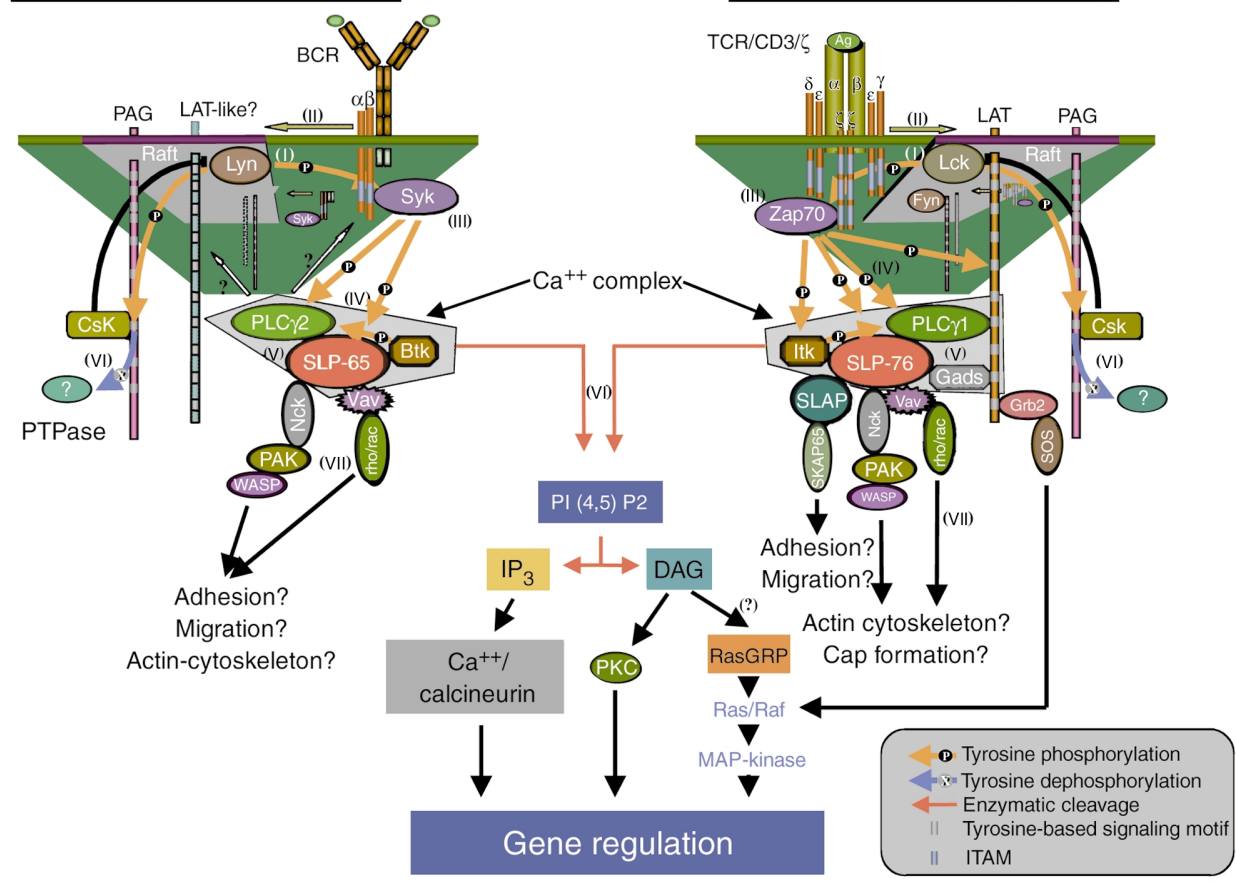




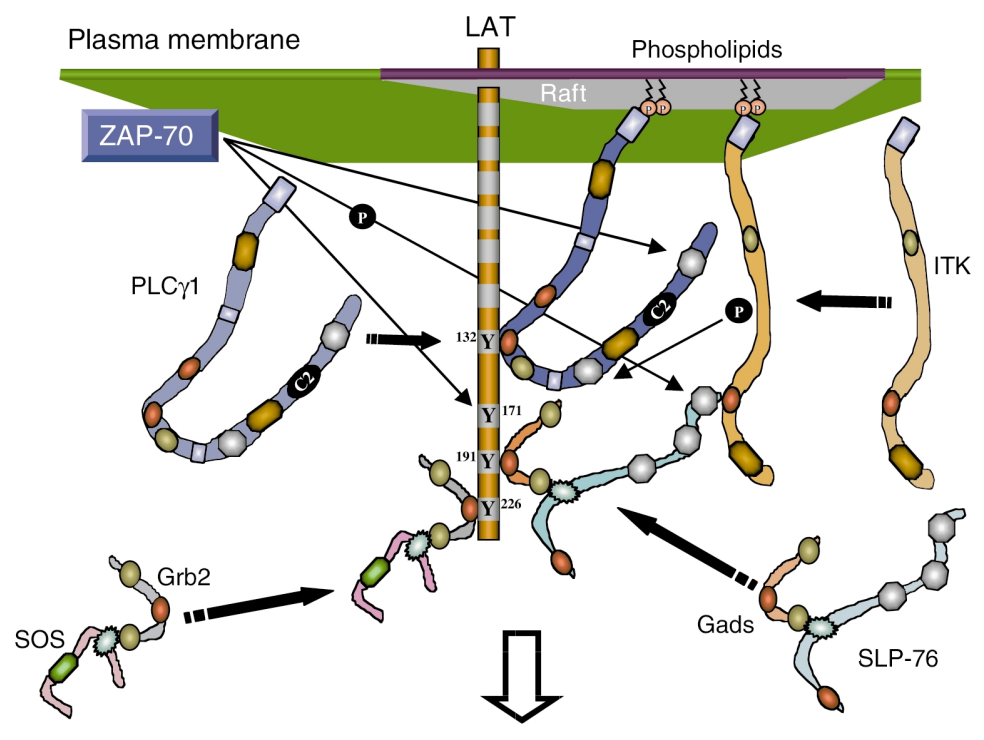

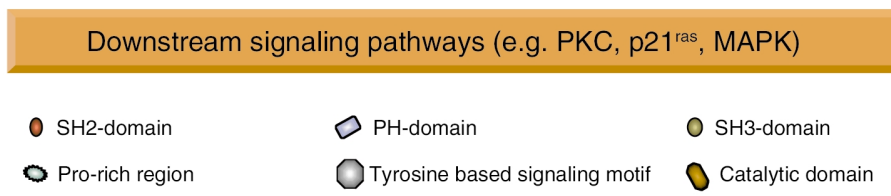

\section{Figure 3}

Detailed illustration of the $\mathrm{Ca}^{+}{ }^{+}$-initiation complex of $\mathrm{T}$ cells. The central unit of this complex is the TRAP LAT, which recruits the Gads/SLP-76 complex and PLC $\gamma 1$ to the lipid rafts after phosphorylation by ZAP-70. The tyrosine kinase Itk binds with its $\mathrm{SH} 2$ domain to phosphorylated SLP-76 and is thus also brought into the vicinity of PLC $\gamma 1$. ZAP-70 and Itk now phosphorylate and activate PCL $\gamma 1$, which, in turn, hydrolyzes $\mathrm{PIP}_{2}$ and generates the secondmessenger molecules DAG and $\mathrm{PIP}_{3}$. Note that Gads and SLP-76 constitutively interact with each other via the $\mathrm{SH} 3$ domain of Gads and a proline-rich region in SLP-76.

signaling molecules and initiate signaling cascades (e.g., formation of the $\mathrm{Ca}^{2+}$-initiation complex) and leading to cellular activation even in the absence of TCR-mediated stimuli. Alternatively, partial activation of Src PTKs could lower the signaling thresholds that are required to activate $\mathrm{T}$ cells. Such "preactivated" $T$ cells could respond to stimuli that, under normal conditions, would rather induce anergy or $\mathrm{T}$ cell unresponsiveness. Conversely, pharmacological inhibition of the PTPase acting on PAG could be beneficial in clinical disorders such

tion (22). This dephosphorylation, which is followed by a partial release of Csk from PAG and from the plasma membrane, could represent one mechanism by which Src PTKs become activated after triggering of the TCR. Candidate protein tyrosine phosphatases (PTPases) that might act on phosphorylated PAG include receptor-type PTPases, such as CD45 or CD148, and cytosolic PTPases, such as SHP-1, SHP-2, and the Csk-associated PTPases PEP and PTP-PEST. Once the relevant enzyme has been defined, it will be important to learn whether TCR triggering stimulates its activity directly or targets it to the subcellular compartment in which PAG resides, namely the lipid rafts discussed below. It will also be important to determine whether the PTPase controls the phosphorylation of more than one TBSM within the PAG cytoplasmic domain, perhaps regulating PAG's association with other $\mathrm{SH} 2$ domain-containing signaling molecules. Considering that PAG carries a total of ten TBSMs in the cytoplasmic domain, such additional interactions seem very likely. An additional function of PAG may be based on the interaction of its C-terminus with cytoplasmic adapter EBP50, which mediates connection to the ezrin-radixin-moesin family proteins and thus to the actin cytoskeleton (25).

The recognition of PAG's ability to recruit Csk to the plasma membrane in resting $\mathrm{T}$ cells and the regulation of this process by a PTPase could provide useful insights into the molecular mechanisms underlying autoimmune diseases. Activities of PTPases are known to be controlled in part by the redox status within the cell (26). Alterations of the redox potential may enhance the activity of the PTPase acting on PAG. This could result in release of Csk from the plasma membrane and, consequently, could enhance the constitutive activity of Src kinases, allowing them to phosphorylate intracellular as autoimmune diseases, transplant rejection, graft-versus-host disease, and even some types of cancer. Analysis of PAG-deficient mice should allow for a deeper understanding of the signaling function of the PAG/Csk complex. It is tempting to speculate that these mice will possess constitutively activated $T$ cells and may be prone to autoimmune disease or lymphomas.

\section{Adapting via membrane rafts}

Recent data suggested that the $\mathrm{Ca}^{2+}$-initiation complex only operates when one key component, LAT, is targeted to particular membrane microdomains called glycosphingolipid-enriched microdomains (GEMs), detergent-resistant membranes (DRMs), detergent-insoluble glycolipid-rich membranes (DIGs), or membrane (lipid) rafts $(4,27)$. Rafts are small areas of the cell membrane that can be distinguished from the rest of the plasma membrane by their unique lipid and protein composition. They are enriched in lipids containing long saturated fatty acid residues (mainly glycosphingolipids), cholesterol, and glycosylphosphatidylinositol-anchored proteins. Several cytoplasmic proteins attach to the inner leaflet of these rafts via covalently bound fatty acid residues (28-30).

Besides PAG and LAT (which are both expressed in T cells as palmitoylated proteins), a number of important signaling molecules are present in rafts. Among these are heterotrimeric $\mathrm{G}$ proteins and certain Src family PTKs possessing $\mathrm{N}$-terminal double acylation. In addition, the cytoplasmic side of rafts is enriched in the signaling phospholipid $\mathrm{PIP}_{2}$, which represents the major substrate for another key component of the $\mathrm{Ca}^{2+}$-initiation complex, PLC $\gamma$. In contrast, most other transmembrane proteins are excluded from rafts. Exceptions include certain receptor molecules, such as CD4, CD8, 
CD44, and CD36, which are palmitoylated on cytoplasmic membrane-proximal cysteine residues.

The integrity of rafts is maintained by lateral interactions of their saturated fatty acid residues, which, compared with the rest of the unsaturated fatty acid-rich membrane, form a more organized, less fluid, liquidordered phase (28). Cholesterol seems to further enhance the stability of these structures due to its interaction with bulky glycosphingolipids (28). Thus, rafts are probably formed spontaneously, simply as a result of phase separation of the specific lipids from the bulk of the membrane. Because it has been notoriously difficult to demonstrate rafts on the surface of living cells, some concern has arisen that rafts are simply in vitro artifacts obtained from membrane lysates. However, several lines of evidence now strongly suggest that rafts exist under physiological conditions (29-33).

Rafts appear to play an essential role in initiation of immunoreceptor signaling (30-33). The currently emerging model holds that the ITAMs of the immunoreceptor-associated signal-transducing components $(\mathrm{CD} 3 \gamma,-\delta,-\varepsilon$, and $-\zeta$, $\operatorname{Ig} \alpha$ and $\operatorname{Ig} \beta$, or the $\gamma$ - and $\beta$-chains of FcERI) remain nonphosphorylated in the absence of external stimuli because the immunoreceptor complexes are excluded from, or perhaps peripherally attached to, rafts where Src PTKs are located. This localization provides an independent mechanism to suppress basal TCR activity, in parallel with the proposed tonic inhibition of Src PTKs by the PAG/Csk complex described above. As a result of their interactions with natural ligands (or after cross-linking by antibodies), aggregates of immunoreceptors form and now merge with rafts (Figure 2), bringing them into proximity with Src PTKs (29).

In T cells, LAT is targeted to the rafts via palmitoylation of two cysteine residues within its cytoplasmic domain (4). Mutation of these palmitoylation sites blocks $\mathrm{T}$ cell activation; or, more precisely, it fails to rescue the signaling defect in the LAT-deficient Jurkat variant, JCaM2 (27). Moreover, elegant studies have shown that experimental targeting of SLP-76 to the rafts restores signaling in this LAT-deficient $\mathrm{T}$ cell line, indicating that one of the major tasks of LAT is to bring SLP-76 to the rafts (7). This hypothesis is supported by the fact that the major substrate of PLC $\gamma 1$, $\mathrm{PIP}_{2}$, is also a major component of the rafts (34). All these findings suggest that rafts are preassembled packages of several molecules involved in initiation of immunoreceptor signaling pathways, ready to be used by triggered immunoreceptors.

A number of questions concerning the nature of rafts and their roles in immunoreceptor signaling and other physiological processes remain. For example, it is not clear whether rafts exhibit a uniform homogeneous composition, or whether there exist different types of rafts. If, indeed, the buoyant fractions collected from the density gradients represent a mixture of diverse raft species differing in their composition, it is possible that some may be enriched in $\mathrm{CD} 4$, others in PAG, and still others in LAT. Preparations of rafts are often heterogeneous in their density, but this has not been systematically explored, and it may reflect only the mixing of membranes originating from different subcellular compartments. Another unsettled question concerns the real size, half-life, dynamics, and cytoskeletal association of rafts on the cell surface under physiological conditions. Recently, rafts were directly visualized (on smooth muscle cells) by singlemolecule microscopy as relatively large $(0.5-2 \mu \mathrm{m})$, stable, and apparently anchored structures (35); other biophysical methods have indicated much smaller dimensions for rafts on leukocytes (36).

Although it is now generally accepted that rafts obtained by detergent solubilization and density gradient ultracentrifugation are not a gross artifact, it remains unclear how closely their composition corresponds to that in native membranes. It seems likely that some of their components are selectively removed during detergent lysis. On the other hand, the use of extremely mild detergent or even mechanical disruption of membranes in the absence of any detergents may produce fragments in which genuine rafts are contaminated by adjacent nonraft areas of the membrane. An important area of further research is the identification, perhaps by proteomics approaches, of additional proteins residing more or less specifically in rafts. For example, there may be additional LAT- and PAG-like TRAPs. The abundance of heterotrimeric $\mathrm{G}$ proteins in rafts is also mysterious. Are they associated there with particular seven-transmembrane domain receptors, or do rafts serve as storage compartments for these important signaling proteins? Finally, the existence of rafts raises the possibility that other types of membrane microdomains exist in which other specific sets of molecules are sequestered. Such hypothetical microdomains might be more difficult to discover if they lacked the pattern of detergent-solubility and -insolubility characteristic of rafts.

\section{Adapting for adhesion and modifications of the actin cytoskeleton}

The CAP SLP-76 apparently plays several roles in the propagation of TCR signals. Not only does this adapter help recruit Itk, thus activating PLC $\gamma 1$ and $\mathrm{Ca}^{2+}$ signaling as discussed above, but it may also regulate lymphocyte adhesion and cytoskeletal changes in activated T cells. Upon T cell activation, SLP-76 interacts via its SH2-domain with the cytosolic adapter SLAP-130 (SLP76 -associated phosphoprotein of $130 \mathrm{kDa}$; also known as FYB, for Fyn-binding protein; or, as recently suggested, ADAP, for adhesion- and degranulation-promoting adapter protein) (37-39). SLAP-130 interacts constitutively with yet another CAP, SKAP55 (Src kinase-associated phosphoprotein of $55 \mathrm{kDa}$ ), in T cells, and with a related signaling protein termed SKAP-HOM or SKAP55R in other hematopoietic cells $(38,40,41)$. The interaction between SLAP-130 and SKAP55/SKAPHOM seems to be of high stoichiometry and to involve a proline-rich region of SLAP-130 and the C-terminal domains of SKAP55 or SKAP-HOM (42).

The function of the SLP-76/SLAP-130/SKAP55 (-HOM) signaling complex is not yet known, but preliminary evidence supports the view that it is involved in regulation of cellular adhesion and migration. One recent study demonstrated that SLAP-130 and SKAP- 
HOM become tyrosine-phosphorylated after plating of macrophages onto fibronectin-coated plates (43). This observation is consistent with the finding that macrophages of SHP-1-deficient mice, which are hyperadhesive, express constitutively phosphorylated SLAP-130 and SKAP-HOM (44). Additional studies revealed that, as in macrophages, triggering of $\beta_{1}$ integrins on human $\mathrm{T}$ cells induces tyrosine phosphorylation of SLAP-130 (45). Changes in $\beta_{1}$-mediated tyrosine phosphorylation of SKAP55 and SKAP-HOM remain to be investigated.

Finally, recent studies demonstrated that a virulence factor of Yersinia enterocolitica, the PTP YopH, dephosphorylates SKAP-HOM and SLAP-130 when expressed in macrophages (the hosts of Yersiniae) $(43,46)$. Interestingly, macrophages infected with Yersiniae are known to adhere and to migrate relatively poorly. The biochemical function of YopH establishes that pathogenic microorganisms can alter immune responses by modifying the signaling properties of adapter proteins. It will be of major interest to determine whether mice lacking the expression of SLAP-130 and SKAP55/SKAP-HOM show an altered sensitivity toward particular bacteria (in particular, those like Listeria monocytogenes, that persist in macrophages) and to investigate whether other microorganisms (e.g., Helicobacter pylori) use similar mechanisms to circumvent the host immune response.

The possibility that the SLP-76/SLAP-130/SKAP55 (-HOM) complex is involved in regulation of cellular adhesiveness might also provide new clues to the propagation of TCR-mediated signals that lead to the high avidity state of integrins. The initial adhesion between antigen-presenting cells (APCs) and T cells is mediated in part by integrins such as lymphocyte function associated antigen-1 (LFA-1), CD 11a, which interacts with its counter-receptor intracellular adhesion molecule-1 (ICAM-1), CD54. On resting lymphocytes, the avidity of LFA-1 toward ICAM-1 is low. However, shortly after engagement of the TCR, the avidity of LFA- 1 becomes strongly upregulated through a process that has been described as "inside-out" signaling. It is thought that the switch of LFA-1 from the low avidity state to the high avidity state is a prerequisite for the activationdependent membrane reconstruction that precedes efficient signal transduction. While intracellular signaling molecules such as cytohesin-1 (a guanine nucleotide exchange factor for small $G$ proteins called ADP-ribosylation factors) play an important role in LFA-1 avidity regulation, it is unclear how the crosstalk is initiated between the TCR and the intracellular signaling cascades, leading to the high avidity state of LFA-1 (47). It is tempting to speculate that the SLP76/SLAP-130/SKAP55(-HOM) complex plays a role in this process. Indeed, the recent description of SLAP130-deficient mice supports this view. Thus, in SLAP130-deficient T cells, TCR triggering apparently fails to induce the high avidity state of integrins $(48,49)$. The phenotypes of SKAP55- and SKAP-HOM-deficient mice are now awaited with curiosity.

Active modification of actin filaments has been shown to be essential for antigen recognition as well as for effective $\mathrm{T}$ cell activation. For example, the initial TCR clusters that form at the nascent immunological synapse are likely to be sites of extensive actin nucleation. Indeed, actin structures in these contact regions can respond rapidly to receptor engagement, and modification of the actin cytoskeleton appears to directly regulate $\mathrm{T}$ cell shape changes associated with TCR clustering and with $\mathrm{T}$ cell migration and contact with APC. An interesting field of research focuses on the question of which intracellular signaling pathways that are initiated after triggering of immunoreceptors induce changes of the cytoskeleton.

Whether members of the $\mathrm{Ca}^{2+}$-initiation complex, such as SLP-76, are involved in TCR-mediated reorganization of the actin cytoskeleton is controversial. A study by Bubeck-Wardenburg et al. suggests that SLP-76 nucleates the formation of a ternary complex comprising SLP-76, Vav, and the adapter protein Nck (the latter two bindings with their SH2 domains to phosphorylated TBSMs of SLP-76) (50). Vav is a guanine nucleotide exchange factor (GEF) for small GTP-binding proteins of the Rho family like Rac1 and CDC42, which mediate actin cytoskeletal rearrangements, whereas Nck mediates associations with downstream effectors of Rac and CDC42, such as the serine threonine kinase $\mathrm{p} 21$-activated kinase 1 (PAK1) or the Wiskott-Aldrich syndrome protein (WASP) $(51,52)$. Activation of PAK1 in turn activates LIM kinase, which phosphorylates an inhibitory residue on cofilin preventing cofilin-mediated disassembly of actin filaments.

It has been proposed that the activation of PAK1 and the subsequent reorganization of the cytoskeleton require simultaneous binding of Vav and Nck to phosphorylated SLP-76, thus bringing Vav in proximity with the GTPases Rac1 and CDC42, which in turn activates PAK1 (50). This model was supported by the finding that overexpression of either molecule enhances TCR-mediated F-actin formation resulting in reorganization of the actin cytoskeleton and cap formation. However, another recent study proposed that while PAK1 activation depends on the presence of ZAP-70, it does not require LAT, SLP-76, and Nck (53). Rather, it was suggested that yet another GEF for Rho GTPases, $\beta$ PIX, is involved in TCR-mediated activation of PAK1. Formation of a ternary complex comprising PAK1, $\beta$ PIX, and the adapter protein PKL, which mediates binding to the integrin-associated protein paxillin, may be required to activate PAK-1. If this is true, this would be the first experimental evidence for a TCR- and PTK-mediated signaling pathway in T cells that acts independently of SLP-76 and LAT. The identification of the signaling components interposed between activated ZAP-70 and the paxillin/PKL/ $\beta$ PIX/PAK1 complex represents one of the major tasks for future research. It is tempting to speculate that this signaling pathway also involves a TRAP, perhaps PAG. In addition, since it has been suggested that SLAP-130 might also be involved in TCR-mediated reorganization of the cytoskeleton, the analysis of SLAP-130-deficient mice might help to shed further light on this matter. 


\section{Adapters and disease}

The analysis of mice made deficient in the expression of adapter proteins has greatly facilitated our understanding of membrane-proximal signaling events in lymphocytes. In addition, recent studies have also identified human diseases that are caused by the loss of expression or function of particular adapter proteins. For example, lack of SLP-65 expression results in an almost complete block of human B cell development at the pro- to pre-B cell stage (54). This causes a severe antibody deficiency of all Ig isotypes - a phenotype that is nearly identical to that of patients suffering from Bruton agammaglobulinemia (or X-linked agammaglobulinemia [XLA]), an immunodeficiency caused by defects in expression or function of the protein tyrosine kinase Btk. The similar B cell defects observed in SLP-65- and Btk-deficient patients provide further support for the observation that SLP-65 and Btk cooperate in the formation of the $\mathrm{Ca}^{2+}$-initiation complex, which is indispensable to mount a humoral immune response (55-57).

SLP-65 is also critically important for the latent persistence of the Epstein-Barr virus (EBV) in human B cells. EBV is the causative agent of infectious mononucleosis and is associated with numerous malignancies including Burkitt lymphoma, Hodgkin disease, and nasopharyngeal carcinoma. Following EBV infection, expression of and signaling from the virally encoded latent membrane protein $2 \mathrm{~A}$ (LMP2A) was found to trigger survival of the infected $B$ cells $(58,59)$. This LMP2A survival signal can substitute for that of the BCR, allowing BCR-negative B cells to colonize peripheral immune organs $(58,59)$. However, transgenic expression of LMP2A in an SLP65-deficient background fails to generate BCR-negative $\mathrm{B}$ cells, showing that SLP-65 is an indispensable downstream effector of LMP2A in vivo. Analysis of the underlying signaling pathways demonstrated that EBV infection of human B cells leads to constitutive association of SLP-65 with Syk, causing constitutive tyrosine phosphorylation of one particular SLP-65 isoform. This triggers permanent activation of some SLP-65-regulated signaling molecules, such as the proto-oncoprotein CrkL (N. Engels et al., unpublished observations).

Another genetically determined disease that arises from the loss of an adapter protein is X-linked lymphoproliferative syndrome (XLP) (60). The most prominent characteristic of XLP is an abnormal response of the immune system against EBV infections, which can eventually result in death from fulminant infectious mononucleosis. The protein affected in XLP is a small CAP called SLAM-associated protein (SAP), which consists of only a single SH2 domain followed by a 26 -amino acid C-terminal tail with no similarity to any known protein. It binds to TBSMs within the cytoplasmic domains of at least two receptors: with high affinity (and independently from tyrosine phosphorylation) to the costimulatory receptor SLAM (CD150) on activated $\mathrm{T}$ cells, and with a lower affinity and in a tyrosine phosphorylation-dependent manner to the 2B4 (CD244) receptor on NK cells (60).
Initially it was proposed that SAP acts as a negative regulatory adapter protein that inhibits signaling via SLAM by preventing binding of the cytosolic PTPase SHP-2 to the receptor. However, more recent data suggest that SAP fulfills a positive regulatory function, facilitating the activation of NK cells through the 2B4 molecule (60). The latter model of SAP function could help to understand the pathophysiology of XLP, since it suggests that one molecular mechanism underlying this disease is a failure to eliminate EBV-infected B lymphoblasts by NK cells. However, additional studies are required to completely understand the function of SAP during B cell and NK cell activation and its role in XLP.

\section{Where do we go next?}

Above, we have focussed on adapter proteins whose functions are already understood to some extent, but many other TRAPs and CAPs for which functional data are scarce are expressed in leukocytes. Clearly, one major goal for the future will be to elucidate the signaling function of all these polypeptides. However, work on the best-studied of the adapter proteins suggests a number of other crucial questions. For example, although their central roles in $\mathrm{T}$ cell activation are well established, it is still not known which intracellular signaling cascades are controlled by the various modular binding domains and TBSMs of SLP-76 or LAT. Reconstitution of SLP-76/LAT-deficient mice with mutated forms of these adapter proteins (or, indeed, CAPs such as SLAP-130 and the SKAPs) could help shed light on the signaling pathways controlled by the various signaling motifs.

Further, in many cases, it remains to be determined which TBSMs in intracellular and transmembrane signaling molecules are required for biological activity. Thus, among the cytoplasmic domains of the four known TRAPs, there are at least 32 TBSMs; the recently identified cytosolic protein $\mathrm{BCAP}$, which is proposed to link the BCR with the phosphatidylinositol 3-kinase pathway, may contain 31 such sites (61). Because these TBSMs may be phosphorylated by more than one class of PTK, the detailed pattern of tyrosine phosphorylation could provide a sensitive indication of the signaling cascades induced by immunoreceptor stimulation.

A recent report (62) offers an intriguing example of how qualitatively different externally applied signals can be translated on the molecular level to produce a specific cellular response. The electrophoretic mobility of tyrosine-phosphorylated TCR $\zeta$ has long been known to differ between $T$ cells stimulated with agonistic peptide ligands and those stimulated with an altered peptide ligand, consistent with differential phosphorylation under these two conditions. This altered migration in SDS-PAGE correlates with the ability of a given peptide ligand to induce activation of ZAP-70. By using polyclonal antisera with specificity for the six tyrosine residues that are expressed in the three ITAMs of $\zeta$, Kersh et al. (62) demonstrated that ITAM phosphorylation and the subsequent activation of ZAP-70 depend on the quality of the peptide binding to the TCR. It seems reasonable to assume that this holds true not only for $\zeta$ but also for the tyrosine phos- 
phorylations of TBSMs within other signaling molecules, especially the various TRAPs.

Most likely, these phosphorylation patterns are regulated not only by the quality of the antigen binding to the immunoreceptor, but also by signals that are transmitted via accessory receptors. The ligands for these accessory receptors are differentially expressed on various cells or environments. Hence, the functional response of a lymphocyte is only partially determined by its genetically fixed programs but is also greatly influenced by the environment in which it is located. Thus, different patterns of tyrosine phosphorylation may arise on membrane-associated and intracellular signaling molecules, depending on the combination of signaltransducing receptors that are activated in a given environment. Under particular circumstances, for instance, environmental factors could make the lymphocyte unresponsive to an antigen that would otherwise activate the cell. One example might be the gut, which harbors millions of $\mathrm{T}$ cells that do not respond toward the multitude of nutritive antigens.

One great challenge in the future will be to visualize different patterns of phosphorylations and/or proteinprotein interactions under various conditions of cellular activation. This could be achieved, for example, by producing antibodies that specifically detect the phosphorylation of individual TBSMs in a given signaling protein and/or by studying the dynamics of proteinprotein interactions in living cells, for instance by using high-resolution microscopy, fluorescence energy transfer analysis, etc. Alternatively, different phosphorylation patterns of a signaling protein could be determined by applying modern mass spectrometry methods, as recently described (63).

In any case, future research aimed at elucidating the function of adapter proteins will require methods that allow analysis of signaling transduction processes without destroying the cells. Such techniques are needed to address other important questions, for example, whether signaling proteins such as LAT or PAG induce distinct signaling cascades in different membrane compartments or in cells that are at different stages of activation or development.

1. Frauwirth, K.A., and Thompson, C.B. 2002. Activation and inhibition of lymphocytes by costimulation. J. Clin. Invest. 109:295-299. DOI:10.1172/JCI200214941.

2. Schaeffer, E.M., and Schwartzberg, P.L. 2000. Tec family kinases in lymphocyte signaling and function. Curr. Opin. Immunol. 12:282-288.

3. Billadeau, D.D., and Leibson, P.J. 2002. ITAMs versus ITIMs: striking a balance during cell regulation. J. Clin. Invest. 109:161-168. DOI:10.1172/JCI200214843.

4. Zhang, W., Trible, R.P., and Samelson, L.E. 1998. LAT palmitoylation: its essential role in membrane microdomain targeting and tyrosine phosphorylation during $\mathrm{T}$ cell activation. Immunity. 9:239-246.

5. Zhang, W., et al. 2000. Association of Grb2, Gads, and phospholipase Cgamma 1 with phosphorylated LAT tyrosine residues. Effect of LAT tyrosine mutations on $\mathrm{T}$ cell antigen receptor-mediated signaling. J. Biol. Chem. 275:23355-23361.

6. Liu, S.K., Fang, N., Koretzky, G.A., and McGlade, C.J. 1999. The hematopoietic-specific adaptor protein gads functions in T-cell signaling via interactions with the SLP-76 and LAT adaptors. Curr. Biol. 9:67-75

7. Boerth, N.J., et al. 2000. Recruitment of SLP-76 to the membrane and glycolipid-enriched membrane microdomains replaces the requirement for linker for activation of T cells in T cell receptor signaling. J. Exp. Med. 192:1047-1058.

8. Wardenburg, J.B., et al. 1996. Phosphorylation of SLP-76 by the ZAP-70 protein-tyrosine kinase is required for T-cell receptor function. J. Biol. Chem. 271:19641-19644.

9. Yoder, J., et al. 2001. Requirement for the SLP-76 adaptor GADS in T cell development. Science. 291:1987-1991.

10. Bunnell, S.C., et al. 2000. Biochemical interactions integrating Itk with the $\mathrm{T}$ cell receptor-initiated signaling cascade. J. Biol. Chem. 275:2219-2230.

11. Liu, K.Q., Bunnell, S.C., Gurniak, C.B., and Berg, L.J. 1998. T cell receptor-initiated calcium release is uncoupled from capacitative calcium entry in Itk-deficient T cells. J. Exp. Med. 187:1721-1727.

12. Baier-Bitterlich, G., et al. 1996. Protein kinase C-theta isoenzyme selective stimulation of the transcription factor complex AP-1 in T lymphocytes. Mol. Cell. Biol. 16:1842-1850.

13. Sun, Z., et al. 2000. PKC-theta is required for TCR-induced NF-kappaB activation in mature but not immature $\mathrm{T}$ lymphocytes. Nature. 404:402-407.

14. Dustin, M.L. 2002. Membrane domains and the immunological synapse: keeping $\mathrm{T}$ cells resting and ready. J. Clin. Invest. 109:155-160. DOI:10.1172/JCI200214842.

15. Monks, C.R., Kupfer, H., Tamir, I., Barlow, A., and Kupfer, A. 1997. Selective modulation of protein kinase C-theta during T-cell activation. Nature. 385:83-86.

16. Roose, J., and Weiss, A. 2000. T cells: getting a GRP on Ras. Nat. Immunol. 1:275-276.

17. Yablonski, D., Kuhne, M.R., Kadlecek, T., and Weiss, A. 1998. Uncoupling of nonreceptor tyrosine kinases from PLC-gamma1 in an SLP-76deficient T cell. Science. 281:413-416.

18. Kurosaki, T., and Tsukada, S. 2000. BLNK: connecting Syk and Btk to calcium signals. Immunity. 12:1-5.

19. Wong, J., Ishiai, M., Kurosaki, T., and Chan, A.C. 2000. Functional complementation of BLNK by SLP-76 and LAT linker proteins. J. Biol. Chem. 275:33116-33122.

20. Ishiai, M., et al. 2000. Involvement of LAT, Gads, and Grb2 in compartmentation of SLP-76 to the plasma membrane. J. Exp. Med. 192:847-856.

21. Engels, N., Wollscheid, B., and Wienands, J. 2001. Association of SLP$65 /$ BLNK with the B cell antigen receptor through a non-ITAM tyrosine of Ig- $\alpha$. Eur. J. Immunol. 31:2126-2134.

22. Brdicka, T., et al. 2000. Phosphoprotein associated with glycosphingolipid-enriched microdomains (PAG), a novel ubiquitously expressed transmembrane adaptor protein, binds the protein tyrosine kinase csk and is involved in regulation of $\mathrm{T}$ cell activation. J. Exp. Med. 191:1591-1604.

23. Kawabuchi, M., et al. 2000. Transmembrane phosphoprotein Cbp regulates the activities of Src-family tyrosine kinases. Nature. 404:999-1003.

24. Cary, L.A., and Cooper, J.A. 2000. Molecular switches in lipid rafts. Nature. 404:945-947.

25. Brdickova, N., et al. 2001. Interaction between two adapter proteins, PAG and EBP50: a possible link between membrane rafts and actincytoskeleton. FEBS Lett. 507:133-136

26. Pani, G., Colavitti, R., Borrello, S., and Galeotti, T. 2000. Redox regulation of lymphocyte signaling. IUBMB Life. 49:381-389.

27. Lin, J., Weiss, A., and Finco, T.S. 1999. Localization of LAT in glycolipidenriched microdomains is required for T cell activation. J. Biol. Chem. 274:28861-28864.

28. Brown, D.A., and London, E. 2000. Structure and function of sphingolipid- and cholesterol-rich membrane rafts. J. Biol. Chem. 275:17221-17224.

29. Simons, K., and Toomre, D. 2000. Lipid rafts and signal transduction. Nat. Rev. Mol. Cell Biol. 1:31-39.

30. Langlet, C., Bernard, A.M., Drevot, P., and He, H.T. 2000. Membrane rafts and signaling by the multichain immune recognition receptors. Curr. Opin. Immunol. 12:250-255.

31. Horejsi, V., et al. 1999. GPI-microdomains: a role in signalling via immunoreceptors. Immunol. Today. 20:356-361.

32. Viola, A., and Lanzavecchia, A. 1999. T-cell activation and the dynamic world of rafts. APMIS. 107:615-623.

33. Katagiri, Y.U., Kiyokawa, N., and Fujimoto, J. 2001. A role for lipid rafts in immune cell signaling. Microbiol. Immunol. 45:1-8.

34. Xavier, R., Brennan, T., Li, Q., McCormack, C., and Seed, B. 1998. Membrane compartmentation is required for efficient $\mathrm{T}$ cell activation. Immu nity. 8:723-732.

35. Schutz, G.J., Kada, G., Pastushenko, V.P., and Schindler, H. 2000. Properties of lipid microdomains in a muscle cell membrane visualized by single molecule microscopy. EMBO J. 19:892-901.

36. Pralle, A., Keller, P., Florin, E.L., Simons, K., and Horber, J.K. 2000. Sphingoloipid-cholesterol rafts diffuse as small entities in the plasma membrane of mammalian cells. J. Cell Biol. 148:997-1008.

37. Musci, M.A., et al. 1997. Molecular cloning of SLAP-130, an SLP-76-associated substrate of the $\mathrm{T}$ cell antigen receptor-stimulated protein tyrosine kinases. J. Biol. Chem. 272:11674-11677.

38. Schraven, B., Marie-Cardine, A., and Koretzky, G. 1997. Molecular analysis of the fyn-complex: cloning of SKAP55 and SLAP-130, two novel 
adaptor proteins which associate with fyn and may participate in the regulation of $\mathrm{T}$ cell receptor-mediated signaling. Immunol. Lett. 57:165-169.

39. da Silva, A.J., et al. 1997. Cloning of a novel T-cell protein FYB that binds FYN and SH2-domain-containing leukocyte protein 76 and modulates interleukin 2 production. Proc. Natl. Acad. Sci. USA. 94:7493-7498.

40. Marie-Cardine, A., et al. 1997. Molecular cloning of SKAP55, a novel protein that associates with the protein tyrosine kinase p59fyn in human Tlymphocytes. J. Biol. Chem. 272:16077-16080.

41. Marie-Cardine, A., Verhagen, A.M., Eckerskorn, C., and Schraven, B. 1998. SKAP-HOM, a novel adapter protein homologous to the FYNassociated protein SKAP55. FEBS Lett. 435:55-60.

42. Marie-Cardine, A., et al. 1998. Molecular interaction between the Fynassociated protein SKAP55 and the SLP-76-associated phosphoprotein SLAP-130. J. Biol. Chem. 273:25789-25795.

43. Black, D.S., Marie-Cardine, A., Schraven, B., and Bliska, J.B. 2000. The Yersinia tyrosine phosphatase YopH targets a novel adhesion-regulated signalling complex in macrophages. Cell. Microbiol. 2:401-414.

44. Timms, J.F., et al. 1999. SHPS-1 is a scaffold for assembling distinct adhesion-regulated multi-protein complexes in macrophages. Curr. Biol. 9:927-930.

45. Hunter, A.J., Ottoson, N., Boerth, N., Koretzky, G.A., and Shimizu, Y. 2000. Cutting edge: a novel function for the SLAP-130/FYB adapter protein in beta 1 integrin signaling and $\mathrm{T}$ lymphocyte migration. J. Immunol. 164:1143-1147.

46. Hamid, N., et al. 1999. YopH dephosphorylates Cas and Fyn-binding protein in macrophages. Microb. Pathog. 27:231-242.

47. Geiger, C., et al. 2000. Cytohesin-1 regulates beta-2 integrin-mediated adhesion through both ARF-GEF function and interaction with LFA-1. EMBO J. 19:2525-2536.

48. Peterson, E.J., et al. 2001. Coupling of the TCR to integrin activation by SLAP-130/Fyb. Science. 293:2263-2265.

49. Griffiths, E.K., et al. 2001. Positive regulation of T cell activation and integrin adhesion by the adapter Fyb/Slap. Science. 293:2260-2263.

50. Bubeck-Wardenburg, J., et al. 1998. Regulation of PAK activation and the
T cell cytoskeleton by the linker protein SLP-76. Immunity. 9:607-616. 51. Li, W., and She, H. 2000. The SH2 and SH3 adapter Nck: a two-gene family and a linker between tyrosine kinases and multiple signaling networks. Histol. Histopathol. 15:947-955.

52. Bustelo, X.R. 2000. Regulatory and signaling properties of the Vav family. Mol. Cell. Biol. 20:1461-1477.

53. Ku, G.M., Yablonski, D., Manser, E., Lim, L., and Weiss, A. 2001. A PAK1PIX-PKL complex is activated by the T-cell receptor independent of Nck, Slp-76 and LAT. EMBOJ. 20:457-465.

54. Minegishi, Y., et al. 1999. An essential role for BLNK in human B cell development. Science. 286:1954-1957.

55. Su, Y.W., et al. 1999. Interaction of SLP adaptors with the SH2 domain of Tec family kinases. Eur. J. Immunol. 29:3702-3711.

56. Hashimoto, S., et al. 1999. Identification of the $\mathrm{SH} 2$ domain binding protein of Bruton's tyrosine kinase as BLNK: functional significance of Btk-SH2 domain in B-cell antigen receptor-coupled calcium signaling. Blood. 94:2357-2364.

57. Kurosaki, T., and Tsukada, S. 2000. BLNK: connecting Syk and Btk to calcium signals. Immunity. 12:1-5.

58. Caldwell, R.G., Wilson, J.B., Anderson, S.J., and Longnecker, R. 1998. Epstein-Barr virus LMP2A drives B cell development and survival in the absence of normal B cell receptor signals. Immunity. 9:405-411.

59. Merchant, M., Caldwell, R.G., and Longnecker, R. 2000. The LMP2A ITAM is essential for providing B cells with development and survival signals in vivo. J. Virol. 74:9115-9124.

60. Morra, M., et al. 2001. X-linked lymphoproliferative disease: a progressive immunodeficiency. Annu. Rev. Immunol. 19:657-682.

61. Okada, T., Maeda, A., Iwamatsu, A., Gotoh, K., and Kurosaki, T. 2000. BCAP: the tyrosine kinase substrate that connects B cell receptor to phosphoinositide 3-kinase activation. Immunity. 13:817-827.

62. Kersh, E.N., Shaw, A.S., and Allen, P.M. 1998. Fidelity of T cell activation through multistep $\mathrm{T}$ cell receptor zeta phosphorylation. Science. 281:572-575.

63. Zhou, H., Watts, J.D., and Aebersold, R. 2001. A systematic approach to the analysis of protein phosphorylation. Nat. Biotechnol. 19:375-378. 